radial cut-down), which was significantly less than femoral DSA minor adverse events $(p=0.0001)$. Radial to femoral conversion rate was $1.2 \%(7 / 607,2$ radial spasm, 2 unsuccessful radial artery access, 3 aberrant right subclavian artery). Femoral to radial conversion rate was 0.3\% (2/635). From March 2019 to February 2020, the proportion of DSA performed via transradial access increased from $36 \%$ to $78 \%$ at our institution.

Conclusion This study demonstrates the learning curves for both TRA and TFA diagnostic cerebral angiograms. Proficiency improves significantly with either type of access, typically requiring between 25-50 procedures. TRA DSA have significantly less access site complications than TFA DSA.

Disclosures M. Tso: None. G. Rajah: None. R. Dossani: None. M. Meyer: None. M. McPheeters: None. K. Vakharia: None. M. Waqas: None. K. Snyder: None. E. Levy: None. A. Siddiqui: None. J. Davies: None.

\section{E-106 EFFICACY OF ASAHI FUBUKI AS A GUIDING CATHETER FOR MECHANICAL THROMBECTOMY: AN INSTITUTIONAL CASE SERIES}

${ }^{1} \mathrm{~L}$ Rinaldo*, ${ }^{2} \mathrm{H}$ Cloft, ${ }^{3} \mathrm{~W}$ Brinjikji. ${ }^{1}$ Neurosurgery, Mayo Clinic, Rochester, MN; ${ }^{2}$ Radiology, Mayo Clinic, Rochester, MN; ${ }^{3}$ Radiology and Neurosurgery, Mayo Clinic, Rochester, MN

\subsection{6/neurintsurg-2020-SNIS.139}

Background Delivery of a guiding catheter to a suitable position is a critical step in performing mechanical thrombectomy (MT) for treatment of acute stroke secondary to large vessel occlusion. Over the past several years, we have employed the Asahi Fubuki (Asahi Intecc Co., LTD, 1976) as a first-line guide catheter for the performance of MT. Herein, we reviewed the treatment outcomes of MT cases in which the Fubuki was utilized to assess the efficacy of this catheter.

Methods Radiology reports of MT for the treatment of acute stroke secondary to large vessel occlusion during which the Fubuki was employed as a guide catheter were retrospectively reviewed. Information on patient characteristics and treatment outcomes was collected. Predictors of failure to deliver the Fubuki to the cervical internal carotid artery (ICA) were identified using logistic regression analysis.

Results There were 124 patients who met the inclusion criteria for analysis. Mean patient age was 77.8 years and a slight majority of patients were female (50.8\%). Large vessel occlusion occurred most often at the M1 segment $(41.1 \%)$ and most occlusions occurred on the patient's right side $(57.5 \%)$. The Fubuki was successfully delivered to the cervical ICA in 121 cases (97.6\%), and the lesion was successfully crossed with the Fubuki as the guide catheter in 119 cases (96.0\%). Herniation of the Fubuki into the aortic arch after delivery to the cervical ICA and cross-over to another guide catheter each occurred once $(0.8 \%)$ in separate cases. Greater than or equal to TICI $2 \mathrm{~B}$ revascularization was achieved in 100 patients $(80.6 \%)$. Median times to lesion crossed and revascularization were 9 and 19 minutes.

Conclusion Our results indicate that the Asahi Fubuki is an effective guide catheter for the performance of MT.

Disclosures L. Rinaldo: None. H. Cloft: None. W. Brinjikji: None.

\section{E-107 PREDICTORS OF DECOMPRESSIVE HEMICRANIECTOMY IN SUCCESSFULLY RECANALIZED PATIENTS WITH ANTERIOR CIRCULATION EMERGENCY LARGE VESSEL OCCLUSION}

${ }^{1} \mathrm{~N}$ Goyal ${ }^{*},{ }^{2} \mathrm{G}$ Tsivgoulis, ${ }^{2} \mathrm{~A}$ Pandhi, ${ }^{2} \mathrm{M}$ Ishfaq, ${ }^{2} \mathrm{~J}$ Goyanes, ${ }^{2} \mathrm{~A}$ Deep, ${ }^{2} \mathrm{D}$ Alsbrook, ${ }^{2} \mathrm{~S}$ Singh, ${ }^{2} \mathrm{G}$ Zaid, ${ }^{3} \mathrm{D}$ Hoit, ${ }^{3} \mathrm{C}$ Nickele, ${ }^{2} \mathrm{~V}$ Inoa, ${ }^{3} \mathrm{D}$ Dornbos, ${ }^{2} \mathrm{~A}$ Alexandrov, ${ }^{3} \mathrm{~A}$ Arthur, ${ }^{1} \mathrm{~L}$ Elijovich. ${ }^{1}$ Neurology and Neurosurgery, UTHSC, Memphis, TN; ${ }^{2}$ Neurology, UTHSC, Memphis, TN; ${ }^{3}$ Neurosurgery, UTHSC, Memphis, TN

\subsection{6/neurintsurg-2020-SNIS.140}

Background and Purpose Mechanical thrombectomy (MT) is demonstrated to improve functional outcome of patients with anterior circulation strokes with emergency large vessel occlusion (ELVO). Despite successful recanalization (SR), a proportion of these patients require decompressive hemicraniectomy (DHC). We aimed to study the predictors of DHC in successfully recanalized anterior circulation ELVO patients.

Methods Consecutive anterior circulation ELVO patients treated with MT during a 6-year period in a tertiary stroke center were evaluated. Only patients with SR (mTICI 2b, 2c or 3) after MT were included in the analysis. Baseline demographic, clinical and procedural variables were compared between the patients who required DHC after successful recanalization vs. who did not. Collaterals for anterior circulation ELVO (ie, poor (collateral score=0) vs. good (collateral score $=1,2$ and 3) were reported using a methodology that has been shown to predict clinical outcome. Good functional outcome was defined as mRS score of $0-2$ at 3 months. Univariable and multivariable logistic regression analyses were used to determine the predictors of DHC.

Results Of 453 successfully recanalized anterior circulation

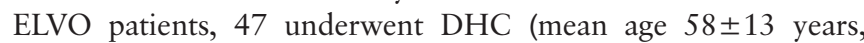
53\% male, median admission NIHSS-score: 17 points, IQR 13-21). The patients in DHC group were younger in age (mean age in years \pm SD; $58 \pm 13$ vs. $65 \pm 15, \mathrm{p}=0.004$ ), had higher admission systolic blood pressure (mmHg: $167 \pm 33$ vs. $155 \pm 30, \mathrm{p}=0.010)$, higher admission diastolic blood pressure (mmHg: $95 \pm 28$ vs. $87 \pm 19, \mathrm{p}=0.019$ ), higher admission blood glucose levels (mg/dl: $170 \pm 88$ vs. $142 \pm 66, \mathrm{p}=0.008$ ), similar median admission NIHSS (17 (IQR: 13-21) vs. 15 (IQR: 1120), $\mathrm{p}=0.087$ ), lower median ASPECTS (9 (IQR: 8-10) vs. 10 (IQR: 9-10), $\mathrm{p}=0.002)$, and poor collaterals on pre-treatment CT angiogram (\% with poor collaterals: $75 \%$ vs. $26 \%$, $\mathrm{p}<0.001)$ compared to those who did not required DHC. The DHC group required more passes during MT (median: 3 (IQR: 3-4) vs. 2 (IQR 1-2), p=0.001), had longer groin puncture to recanalization time (mean: $74 \pm 32$ vs. $55 \pm 35$, $\mathrm{p}<0.001$ ), while no difference was seen in symptoms onset to groin puncture time (mean: $313 \pm 166$ vs. $304 \pm 202, p=0.784$ ) compared to the non-DHC group. The patient in DHC group tended to have a higher $\mathrm{sICH}$ rate $(15 \%$ vs. $7 \%, \mathrm{p}=0.20)$, higher 3 months mortality $(41 \%$ vs. $16 \%, p=0.001)$ and lower rates of good functional outcome at 3 months $(26 \%$ vs. $58 \%, \mathrm{p}=0.001)$. In multivariable model after adjusting for various confounders, higher blood glucose levels on admission (OR per $1 \mathrm{mg} / \mathrm{dL}$ increase: 1.01; 95\% CI: 1.00-1.01, $\mathrm{p}=0.031$ ), poor collaterals on CT angiography (OR: 0.13 ; 95\% CI: $0.05-0.32, \mathrm{p}<0.001)$, and higher number of passes during MT (OR per 1 pass increase: 4.84; 95\% CI: 3.09$7.58, \mathrm{p}<0.001$ ) emerged as independent predictors of $\mathrm{DHC}$ in successfully recanalized ELVO patients.

Conclusion Higher blood glucose levels on admission, poor collateral pattern on CT angiography and higher number of 
passes during MT were independently associated with DHC in anterior circulation EVLO patients achieving SR at the end of MT. Of these, admission glucose levels and number of passes during MT are potentially modifiable factors.

Disclosures N. Goyal: None. G. Tsivgoulis: None. A. Pandhi: None. M. Ishfaq: None. J. Goyanes: None. A. Deep: None. D. Alsbrook: None. S. Singh: None. G. Zaid: None. D. Hoit: None. C. Nickele: None. V. Inoa: None. D. Dornbos: None. A. Alexandrov: None. A. Arthur: None. L. Elijovich: None.

\section{E-108 LONG TERM CLINICAL AND ANGIOGRAPHIC OUTCOME WITH THE INTRACRANIAL STENT FOR SYMPTOMATIC INTRACRANIAL STENOSIS}

'S Park*, ${ }^{2} \mathrm{~S}$ Seo, 'J Kim. 'Neurosurgery, Gangnam Severance Hospital, Yonsei University, Seoul, Korea, Republic of, ${ }^{2}$ Neuroradiology, Gangnam Severance Hospital, Yonsei University, Seoul, Korea, Republic of

\subsection{6/neurintsurg-2020-SNIS.141}

Background and Aims The risk of ischemic stroke from a symptomatic stenotic intracranial artery is high despite best medical therapy (BMT). Clinicians have increasingly turned to percutaneous transluminal angioplasty and stenting (PTAS) over the last decades as an alternative therapy in high-risk patient with symptomatic ICAS. We evaluated long-term clinical outcome and restenosis rate with atherosclerotic intracranial stenosis using the Wingspan self-expanding nitinol stent system.

Methods The patients treated with the Wingspan in two medical center from January 2010 to December 2016 were enrolled. Target patients were affected by high-grade, symptomatic, intracranial atherosclerotic lesions, were on antithrombotic therapy and at high stroke risk. Follow up DSA was performed at 1-year. After that, image follow up was done by CTA, MRA or DSA. The patients were followed up at least 3 years.

Results 43 patients treated with Wingspan stent for symptomatic intracranial stenosis were enrolled in this study. The frequency for any stroke or death within 30-days was $7 \%$. The frequency of any stroke or death after 30-day was also $7 \%$ at mean 58.7 months follow up period. The frequency of $>50 \%$ restenosis on 3 -year follow up image was $18 \%(n=8 / 43)$. There was complete occlusion in $9 \%(n=4 / 43)$. Reballooning was done in the other 4 patients but final occlusion was seen in 2 patients.

Conclusions The use of Wingspan stent in patient with $>50 \%$ symptomatic intracranial stenosis is associated with good long term clinical outcome. Long term restenosis rate was not high and asymptomatic in most patients. The incidence of symptomatic infarct was low among the restenosis patients.

Disclosures S. Park: None. S. Seo: None. J. Kim: None.

\section{E-109 NATIONAL TRENDS SHOW IMPROVEMENT OF CLINICAL OUTCOME WITH ISCHEMIC STROKE THROUGH ADVANCES IN ENDOVASCULAR THERAPY}

${ }^{1} \mathrm{~K}$ Seo*, ${ }^{2} \mathrm{~S}$ Suh, ${ }^{3} \mathrm{~K}$ Lee, ${ }^{4} \mathrm{M}$ Kang. ${ }^{1}$ Neurology, National Health Insurance Service Ilsan Hospital, Goyang, Korea, Republic of; ${ }^{2}$ Radiology, Gangnam Severance Hospital, Yonsei University College of Medicine, Seoul, Korea, Republic of; ${ }^{3}$ Neurology, Gangnam Severance Hospital, Yonsei University College of Medicine, Seoul, Korea, Republic of; ${ }^{4}$ Institute of Health Insurance and Clinical Research, National Health Insurance Service Ilsan Hospital, Goyang, Korea, Republic of

10.1136/neurintsurg-2020-SNIS. 142
Background and Purpose Endovascular therapy (ET) of ischemic stroke with large vessel occlusion has been proved effective by clinical trials and reported with good outcomes in real clinical settings since 2015. We aim to determine national trends of clinical outcome in ET and compare the outcomes before and after mechanical thrombectomy.

Methods We obtained all data from the nationwide database of Health Insurance Review \& Assessment Service from 2008 to 2017. The patients with ischemic stroke who received ET were identified using International classification of disease-codes and several codes regarding to ET. In evaluating prognosis of patients receiving ET, good outcome was defined as discharge to home and poor outcomes as cerebral hemorrhage, physical disabilities and death. We analyzed the time points of ET to determine the factors affecting the prognosis. The study period was divided into three periods: 1.when stent-retriever was not used (non-ET period), 2.when it was used for off-label and non-reimbursement (transitional period), and 3.when it was reimbursed (ET period).

Results A total of 15,589 patients who were received ET between January 2008 and December 2017 were analyzed. Of the total subjects, 5,512 patients (35.4\%) received intravenous tPA. 3,028 were treated at non-ET period, 4,113 were treated at transitional period and 5,360 were treated at ET period. The rate of home discharge was $35.9 \%, 39.7 \%$ and $41.7 \%$ respectively, which was statistically significant $(\mathrm{p}<0.0001)$. 3-months mortality were $25.7 \%, 22.0 \%, 19.5 \%$ $(\mathrm{p}<0.0001)$, and 1-year mortality were $34.5 \%, 30.1 \%$ and $26.4 \%(p<0.0001)$. There was a decrease in the likelihood of death (hazard ratio $0.693,95 \%$ CI $0.645-0.744$ ) in ET period. The rate of disability was $33.6 \%, 26.5 \%$ and $23.4 \%$ $(\mathrm{p}<0.0001)$.

Conclusions The nationwide health insurance data showed clinical outcomes of patients with ischemic stroke after ET were significantly better in the ET period in Korea.

Disclosures K. Seo: None. S. Suh: None. K. Lee: None. M. Kang: None.

\section{E-110 PATIENT COUNSELING ON SMOKING CESSATION FOLLOWING ISCHEMIC STROKE}

${ }^{1} \mathrm{~K}$ Swafford*, ${ }^{1} \mathrm{~J}$ Frank, ${ }^{2} \mathrm{~J}$ Roberts, ${ }^{1} \mathrm{~A}$ Trout, ${ }^{3} \mathrm{~S}$ Grupke, ${ }^{4} \mathrm{~K}$ Pennypacker, ${ }^{3} \mathrm{~J}$ Fraser. ${ }^{1}$ Neurology, University of Kentucky, Lexington, $K Y ;{ }^{2}$ Neurosurgery, Neuroscience, University of Kentucky, Lexington, $K Y$; ${ }^{3}$ Neurosurgery, University of Kentucky, Lexington, $K Y_{\text {; }}$ ${ }^{4}$ Neurology, Neuroscience, University of Kentucky, Lexington, $K Y$

\subsection{6/neurintsurg-2020-SNIS. 143}

Introduction/Purpose Ischemic stroke is the second leading cause of death in the United States. Smoking accelerates the onset of stroke, on average, by ten years. We sought to analyze the effects of smoking status following endovascular treatment (EVT) for large vessel occlusion in patients with acute ischemic stroke. Through our previously published Blood And Clot Thrombectomy Registry And Collaboration (BACTRAC) stroke tissue bank (ClinicalTrials.gov: NCT03153683), we aimed to determine the effects of smoking on the change in National Institutes of Health Stroke Scale (NIHSS) score, infarct volume, and cerebral edema volume. 